PROCEEDINGS OF THE

AMERICAN MATHEMATICAL SOCIETY

Volume 132, Number 5, Pages 1351-1354

S 0002-9939(03)07363-5

Article electronically published on December 23, 2003

\title{
LOCAL UNIQUENESS FOR THE FIXED ENERGY FIXED ANGLE INVERSE PROBLEM IN OBSTACLE SCATTERING
}

\author{
PLAMEN STEFANOV AND GUNTHER UHLMANN
}

(Communicated by David S. Tartakoff)

\begin{abstract}
We prove local uniqueness for the inverse problem in obstacle scattering at a fixed energy and fixed incident angle.
\end{abstract}

We consider the inverse problem of determining a sound-soft obstacle in $\mathbf{R}^{n}$, $n \geq 2$, from its scattering amplitude at a fixed incident direction $\theta \in S^{n-1}$ and a fixed energy $k>0$. This is a formally determined inverse problem, since the data depends on the same number of variables, $n-1$, as does the object we want to recover.

The purpose of this note is to give a simple proof of local uniqueness for this problem. Roughly speaking, we show that if two domains are close to a given obstacle, in a precise sense described below, and have the same scattering amplitude at a fixed angle and fixed energy, then they must be the same. Previously, it was shown in [CS] that local uniqueness holds for small obstacles. The Fréchet derivative of the nonlinear map from the domain to the scattering amplitude at fixed energy and angle was computed in $[\mathrm{P}$, and one can easily show that it is injective. However, this does not imply a local result, since we cannot directly apply the implicit function theorem.

The proof of our result follows by using the arguments of Schiffer's well-known proof, presented in $[\mathrm{LP}$, of uniqueness when given all incident directions, and the Poincaré inequality. By obstacles, we mean compact subsets of $\mathbf{R}^{n}$ with $C^{2}$ boundary and connected complement.

The scattering amplitude $A_{\mathcal{O}}(k, \theta, \omega)$ related to an obstacle $\mathcal{O}$ is defined as follows. For $k>0, \theta \in S^{n-1}$, we define the scattering solution $u(x, \theta, k)$ as the solution to the boundary value problem (see, e.g., [CK]

$$
\left\{\begin{aligned}
\left(-\Delta-k^{2}\right) u & =0, \quad \text { in } \mathbf{R}^{n} \backslash \mathcal{O}, \\
\left.u\right|_{\partial \mathcal{O}} & =0,
\end{aligned}\right.
$$

Received by the editors August 19, 2002.

2000 Mathematics Subject Classification. Primary 35R30; Secondary 81U40, 35P25.

The first author was partly supported by NSF Grant DMS-0196440 and MSRI.

The second author was partly supported by NSF Grant DMS-007048 and a John Simon Guggenheim fellowship. Both authors would like to thank the hospitality of the Mathematical Sciences Research Institute where part of this work was done.

(C)2003 American Mathematical Society 
such that $u=e^{i k \theta \cdot x}+v$, with $v$ satisfying the Sommerfeld outgoing condition at infinity: $(\partial / \partial r-i k) v=O\left(r^{-(n+1) / 2}\right)$, as $r=|x| \rightarrow \infty$. Then

$$
v(x, \theta, k)=e^{i k \theta \cdot x}+\frac{e^{i k r}}{r^{(n-1) / 2}} A_{\mathcal{O}}\left(k, \theta, \frac{x}{r}\right)+O\left(\frac{1}{r^{(n+1) / 2}}\right), \quad \text { as } r=|x| \rightarrow \infty .
$$

The function $A_{\mathcal{O}}(k, \theta, \omega)$ is the scattering amplitude related to $\mathcal{O}$.

It is known that Schiffer's proof implies uniqueness if $A_{\mathcal{O}}$ is known for all $\omega$, fixed $k_{0}>0$, and $N$ incident directions $\theta$; or for all $\omega$, fixed $\theta_{0}$, and $N$ frequencies $k \leq k_{0}$, where $N$ is greater than the number of the Dirichlet eigenvalues $k^{2} \leq k_{0}^{2}$ of the Laplacian in a ball containing the obstacles. In particular, as mentioned above, this implies uniqueness at a fixed $\theta_{0}$ and a fixed $k_{0}$ for all obstacles contained in a ball with sufficiently small radius $R$. In the $3 \mathrm{D}$ case, the condition is given by $k_{0} R<\pi$. We refer to [CK], $\mathbf{I},[\mathrm{KK}$, , $\mathrm{CS}]$ for details and references.

In what follows, $\omega_{n}$ is the volume of the unit ball in $\mathbf{R}^{n}$ (not to be confused with the outgoing direction $\omega$ ); in particular, $\omega_{3}=4 \pi / 3$.

Our main result is the following.

Theorem 1. Fix $k_{0}>0, \theta_{0} \in S^{n-1}$. Let $\mathcal{O}_{-} \subset \mathcal{O}_{+}$be two obstacles and assume that $\operatorname{Vol}\left(\mathcal{O}_{+} \backslash \mathcal{O}_{-}\right)<\omega_{n} k_{0}^{-n}$. Let $\mathcal{O}_{-} \subset \mathcal{O}_{j} \subset \mathcal{O}_{+}, j=1,2$, be two other obstacles and assume that $A_{\mathcal{O}_{1}}\left(k_{0}, \theta_{0}, \omega\right)=A_{\mathcal{O}_{2}}\left(k_{0}, \theta_{0}, \omega\right)$. Then $\mathcal{O}_{1}=\mathcal{O}_{2}$.

In particular, for any fixed obstacle $\mathcal{O}$, and fixed $k_{0}>0, \theta_{0}$, any small enough perturbation of the boundary gives an obstacle with different scattering amplitude.

More precisely, there exists $\varepsilon=\varepsilon\left(\mathcal{O}, k_{0}, \theta_{0}\right)>0$ such that if $\partial \mathcal{O}_{1}$ is given in boundary normal coordinates $\left(x^{\prime}, x_{n}\right) \in \partial \mathcal{O} \times(-\delta, \delta)$ by $x_{n}=f\left(x^{\prime}\right)$ with $\left|f\left(x^{\prime}\right)\right| \leq \varepsilon$, $\forall x^{\prime}$, then $A_{\mathcal{O}_{1}}\left(k_{0}, \theta_{0}, \omega\right)=A_{\mathcal{O}}\left(k_{0}, \theta_{0}, \omega\right)$ implies $\mathcal{O}_{1}=\mathcal{O}$. We would like to emphasize that this is different from the uniqueness for obstacles with small diameters mentioned above.

In Theorem 1 and Proposition 1 below, we do not impose smallness assumptions on $k_{0}$ or on the diameters of the obstacles. We prove unconditional local uniqueness at fixed $k_{0}, \theta_{0}$ near any obstacle.

Let $\Omega_{\text {ext }}$ be the connected unbounded component of $\mathbf{R}^{n} \backslash\left(\mathcal{O}_{1} \cup \mathcal{O}_{2}\right)$. Set $\Omega_{\text {int }}=$ $\mathbf{R}^{n} \backslash \bar{\Omega}_{\text {ext }}$. Then $\bar{\Omega}_{\text {int }} \supset \mathcal{O}_{1} \cup \mathcal{O}_{2}$. Note that $\Omega_{\text {int }}$ is an open set that contains the interior of $\mathcal{O}_{1} \cup \mathcal{O}_{2}$ as well as all components of $\mathbf{R}^{n} \backslash\left(\mathcal{O}_{1} \cup \mathcal{O}_{2}\right)$ disconnected from infinity.

Theorem 1 follows from the following.

Proposition 1. Let $\mathcal{O}_{1}$ and $\mathcal{O}_{2}$ be two obstacles. Assume that for the corresponding scattering amplitudes we have

$$
A_{\mathcal{O}_{1}}\left(k_{0}, \theta_{0}, \omega\right)=A_{\mathcal{O}_{2}}\left(k_{0}, \theta_{0}, \omega\right)
$$

for a fixed $\theta_{0} \in S^{n-1}$, fixed $k_{0}>0$ and all $\omega \in S^{n-1}$. If

$$
\operatorname{Vol}\left(\Omega_{\text {int }} \backslash \mathcal{O}_{i}\right)<\omega_{n} k_{0}^{-n}, \quad i=1,2,
$$

then $\mathcal{O}_{1}=\mathcal{O}_{2}$.

Our argument is based on an estimate of the first eigenvalue of the Dirichlet Laplacian in a bounded domain.

Lemma 1. Let $k^{2}$ be a Dirichlet eigenvalue of $-\Delta$ in the bounded domain $G$. Then

$$
\omega_{n} \leq k^{n} \operatorname{Vol}(G) .
$$


Proof. We use the Poincaré inequality in the form presented in [GT:

$$
\|u\| \leq\left(\frac{\operatorname{Vol}(G)}{\omega_{n}}\right)^{1 / n}\|\nabla u\|, \quad \text { for any } u \in H_{0}^{1}(G) .
$$

Let $u$ be a normalized eigenfunction corresponding to $k^{2}$. Then $\|\nabla u\|=k$ and $u \in H_{0}^{1}(G)$. Applying (2), we get

$$
1 \leq k\left(\frac{\operatorname{Vol}(G)}{\omega_{n}}\right)^{1 / n}
$$

which implies the lemma.

Proof of Proposition 1. The proof is a combination of Schiffer's idea and Lemma 1

Let $u_{j}(x, \theta, k)$ be the scattering solution related to $\mathcal{O}_{j}, j=1,2$. By a wellknown argument based on Rellich's lemma, $A_{\mathcal{O}_{1}}\left(k_{0}, \theta_{0}, \omega\right)=A_{\mathcal{O}_{2}}\left(k_{0}, \theta_{0}, \omega\right)$ implies that $u_{1}\left(x, \theta_{0}, k_{0}\right)=u_{2}\left(x, \theta_{0}, k_{0}\right)$ for all $x$ outside a ball containing $\mathcal{O}_{1} \cup \mathcal{O}_{2}$. We know that $u_{1}$ and $u_{2}$ solve

$$
\left\{\begin{aligned}
\left(-\Delta-k_{0}^{2}\right) u_{j} & =0, \quad \text { in } \mathbf{R}^{n} \backslash \mathcal{O}_{j} \\
\left.u_{j}\right|_{\partial \mathcal{O}_{j}} & =0 .
\end{aligned}\right.
$$

Then by analytic continuation, $u_{1}=u_{2}$ on $\partial \Omega_{\text {ext }}$.

Suppose that $\mathcal{O}_{1} \neq \mathcal{O}_{2}$. Then for $j=1$ or $j=2, \Omega_{\text {int }} \backslash \mathcal{O}_{j}$ is an open nonempty set. Suppose that this happens for $j=1$. Let $G$ be any connected component of $\Omega_{\text {int }} \backslash \mathcal{O}_{1}$. Then $u_{1}=0$ on $\partial G$, and therefore $\left.u_{1}\right|_{G} \in H_{0}^{1}(G)$. Since $\partial G$ may not be smooth, the latter needs some justification. This was done in [CK] by approximating $u_{1}$ by a sequence $u_{1, n} \in C_{0}^{\infty}(G)$; see [CK, Theorem 5.1 and Lemma 3.8]. Therefore, $u_{1}$ solves the problem

$$
\left\{\begin{aligned}
\left(-\Delta-k_{0}^{2}\right) u_{1} & =0, \text { in } G \\
\left.u_{1}\right|_{G} & \in H_{0}^{1}(G)
\end{aligned}\right.
$$

Moreover, $u_{1}$ is not identically equal to zero in $G$, because it is a real analytic function in the domain $\mathbf{R}^{n} \backslash \mathcal{O}_{1}$ not vanishing for large $x$. Thus $k_{0}^{2}$ is a Dirichlet eigenvalue of the Laplacian in $G$.

By Lemma1 $\omega_{n} k_{0}^{-n} \leq \operatorname{Vol}(G) \leq \operatorname{Vol}\left(\Omega_{\mathrm{int}} \backslash \mathcal{O}_{1}\right)$. This contradicts our assumption (11), which proves the proposition. Note that in (1) , we can actually replace $\Omega_{\text {int }} \backslash \mathcal{O}_{j}$ by the biggest (in terms of volume) connected component of this set.

Proof of Theorem [1. We claim that the open sets $\Omega_{\text {int }} \backslash \mathcal{O}_{j}$ are included in $\mathcal{O}_{+} \backslash \mathcal{O}_{-}$.

To prove that, note that $\Omega_{\text {int }} \backslash \mathcal{O}_{1}$, for example, is a union of the interior of $\mathcal{O}_{2} \backslash \mathcal{O}_{1}$ and all bounded components of $\mathbf{R}^{n} \backslash\left(\mathcal{O}_{1} \cup \mathcal{O}_{2}\right)$. We only need to show that any such component is in $\mathcal{O}_{+} \backslash \mathcal{O}_{-}$. Assume that there is a point $x_{0}$ in such a component with $x_{0} \notin \mathcal{O}_{+} \backslash \mathcal{O}_{-}$. Clearly, $x_{0} \notin \mathcal{O}_{+}$. Then we can connect $x_{0}$ and infinity with a continuous curve lying outside $\mathcal{O}_{+}$, because $\mathcal{O}_{+}$is an obstacle. This curve is in $\mathbf{R}^{n} \backslash\left(\mathcal{O}_{1} \cup \mathcal{O}_{2}\right)$, and this contradicts the assumption that $x_{0}$ is in a bounded component of this set. This proves the inclusion, and the theorem now follows from Proposition 1

\section{REFERENCES}

[CK] D. Colton and R. Kress, Inverse Acoustic and Electromagnetic Scattering Theory, 2nd edition, Applied Mathematical Sciences, Vol. 93, Springer-Verlag, Berlin, 1998. MR 99c:35181

[CS] D. Colton and B. D. Sleeman, Uniqueness theorems for the inverse problem of acoustic scattering, IMA J. Appl. Math. 31(3) (1983), 253-259. MR 85e:76044 
[GT] D. Gilbarg and N. Trudinger, Elliptic Partial Differential Equations of Second Order, Grundlehren der Mathematischen Wissenschaften, Vol. 224, Springer-Verlag, Berlin, 1977. MR 57:13109

[I] V. Isakov, Inverse problems for partial differential equations, Appl. Math. Sci., 127, Springer-Verlag, New York, 1998. MR 99b:35211

[KK] A. Kirsch and R. Kress, Uniqueness in inverse obstacle scattering, Inverse Problems 9 (1993), 285-299. MR 94e:35143

[LP] P. Lax and R. Phillips, Scattering Theory, Academic Press, 1967. MR 36:530

[P] R. Potthast, Fréchet differentiability of boundary integral operators in inverse acoustic scattering, Inverse Problems 10 (1994), 431-447. MR 95c:35268

Department of Mathematics, Purdue University, West Lafayette, Indiana 47907

E-mail address: stefanov@math.purdue.edu

Department of Mathematics, University of Washington, Seattle, Washington 98195

E-mail address: gunther@math. washington.edu 